\title{
Relictos de vegetación en tramos fluviales intermitentes en el sector medio de las cuencas de Sama, Caplina y Hospicio, Tacna, Perú
}

\author{
Relics of vegetation in intermittent river sections in the middle sector of the basins of Sama, \\ Caplina and Hospicio, Tacna, Peru
}

\author{
${ }^{1, a}$ Syntia Huisa \\ ${ }^{1, b}$ Yarina Flores \\ ${ }^{1, \mathrm{c}}$ Tonantzin Valdez \\ ${ }^{1, \mathrm{~d}}$ Dayana Condori
}

\author{
${ }^{1, \mathrm{e}}$ Josué Pumasupa \\ 1,f Zulma Huamantalla \\ ${ }^{1, g}$ Jackeline Arias \\ ${ }^{1,2, \mathrm{~h}}$ Alex Yaja
}

\begin{abstract}
ARTÍCULO ORIGINAL
${ }^{1}$ Universidad Nacional Jorge Basadre Grohmann. Semillero en Limnología, Facultad de Ciencias. Tacna, Perú ${ }^{2}$ Universidad Nacional Jorge Basadre Grohmann. Herbario Takana, Facultad de Ciencias. Tacna, Perú. E-mail: ayajac@unjbg.edu.pe

aORCID: 0000-0002-2290-5068

'ORCID: 0000-0002-6319-731X

'ORCID: 0000-0003-4461-9473

dORCID 0000-0002-6808-2159

'ORCID: 0000-0002-8040-5547

f ORCID: 0000-0001-8053-2477

${ }^{g}$ ORCID: 0000-0003-1816-8090

hORCID: 0000-0001-9996-6582
\end{abstract}

Palabras clave: Correlación espacial, índices morfométricos, NDVI, precipitación.

Keywords: Spatial correlation, morphometric indices, NDVI, precipitation.

Información adicional

Presentado: $3 / 11 / 2020$

Aprobado: 18/12/2020

\section{RESUMEN}

El objetivo de este trabajo consistió en caracterizar los factores topográficos, la variabilidad del vigor vegetal y la relación con la precipitación a nivel de cuenca de una serie de bosques ribereños aislados en la zona desértica del piedemonte andino-costero en las cuencas de Sama, Caplina y Hospicio. Se calcularon tres índices morfométricos para caracterizar el terreno en el que se desarrollan estos parches de vegetación ribereña. Además, la serie histórica de vigor vegetal (NDVI entre 1985 y 2011) se comparó con datos de precipitación extraídos de la base grillada PISCO. Encontramos que estos bosques se desarrollan en zonas con una pendiente media a baja (entre $5^{\circ}$ a $15^{\circ}$ ), el valor del índice de protección morfométrica se ubica entre 0.16 y 0.30 unidades (alta protección) y el valor de la humedad topográfica está entre 12 a 18 unidades. En cuanto a la relación entre el NDVI y la precipitación, la mayor influencia es ejercida por las lluvias en la zona de recarga en la cuenca (cabeceras). Por las características del terreno en el que se desarrollan y la relación entre vegetación y clima se deduce que estos son ecosistemas dependientes de agua subterránea.

\section{ABSTRACT}

The objective of this work was to characterize the topographic factors, the variability of the vegetation vigor and the relation with precipitation at basin level of a series of isolated riparian forests in the desert zone of the AndeanCoastal foothills in the Sama, Caplina and Hospicio basins. Three morphometric indices were calculated to characterize the terrain morphometry in which those patches of riparian vegetation were stablished. In addition, the historical series of plant vigor (NDVI between 1985 and 2011) was compared with the precipitation data extracted from the PISCO database. It was found that these forests develop in areas with a medium to low slope (between $5^{\circ}$ to $15^{\circ}$ ), the value of the morphometric protection index is between 0.16 and 0.30 units (high protection) and the value of humidity topographic is between 12 to 18 units. Regarding the relationship between NDVI and precipitation, the greatest influence is exerted by the rains in the basin recharge zone (headwaters). Due to the characteristics of the terrain in which they develop and the relation between vegetation and climate, it can be deduced that those ecosystems are dependent on groundwater. 


\section{INTRODUCCIÓN}

En regiones áridas y semi-áridas de todo el mundo es posible encontrar sectores con características de protección geográfica que mantienen agua disponible durante todo el año, e incluso forman tramos de ríos intermitentes (Datry et al., 2017). En dichos ecosistemas la disponibilidad del recurso hídrico (RRHH) está restringida y - por tanto - este constituye el elemento limitante para el desarrollo de toda la comunidad, incluyendo al ser humano (Arnold et al., 2016). Por su carácter único y por constituir espacios que ofrecen un recurso escaso, estos ríos intermitentes representan refugios de biodiversidad que contribuyen de manera importante al acervo genético regional (Wang \& D'Odorico, 2008). A pesar de su importancia, la información generada sobre estos ecosistemas es aún escasa, por lo que no se logra comprender detalladamente su ecología (Leigh et al., 2016).

Generalmente, se trataría de ecosistemas dependientes de aguas subterráneas, en los que se experimentan eventos de flujo incrementado asociados a períodos de lluvias intensas que recarguen los acuíferos regionales, y una posterior desecación de muchos tramos en la temporada seca con la permanencia de estanques aislados (Bunn et al., 2006; Stanley et al., 1997). La ecología acuática está determinada por la frecuencia, duración y magnitud de los sucesos de inundación - desecación que controlan y mantienen la diversidad biológica con una dinámica metapoblacional muy marcada (Bunn et al., 2006). Las condiciones generales de aislamiento promueven una alta tasa de desarrollo de algas y el ingreso de materia y energía a la cadena alimentaria, lo que permite la presencia de una comunidad rica de zooplancton que sirve de alimento para muchos consumidores (Bunn et al., 2003, 2006). Pero esto mismo vuelve limitantes las condiciones fisicoquímicas del medio, lo que obliga a la comunidad a adaptarse a un ambiente restrictivo (Kalogianni et al. 2017; Von Schiller et al., 2017).

Contigua a estos ecosistemas acuáticos, se desarrolla una vegetación ribereña vigorosa que también depende de la humedad disponible, proporcionando servicios ecosistémicos como hábitat y fuente de recursos para la comunidad biótica terrestre, protección (sombra) para el ecosistema acuático y mantenimiento de la vitalidad ecológica (Stromberg \& Merritt, 2016). La profundidad del agua subterránea y la permanencia del flujo provocan variaciones en la composición de especies y el desarrollo, densidad y estructura de estas formaciones vegetales (Stromberg \& Merritt, 2016). Finalmente, muchos de estos tramos suelen ser eficientemente utilizados por la población humana para desarrollar actividades pecuarias, con un uso eficiente del recurso hídrico (Kaletová et al., 2019). Un ejemplo de esto último lo representa el Área Prioritaria de Conservación Regional Cinto, un río intermitente que es afluente por la margen derecha (Norte) del río Locumba donde el escaso recurso hídrico es usado para el desarrollo de actividades agrícolas (O.R. No 012-2015-CR/GOB.REG.TACNA).

Para el conocimiento de los autores, no existen estudios específicos con respecto a la ecología en tramos fluviales intermitentes en el desierto costero del Perú (por lo tanto, no se cuenta con información para la región Tacna). Únicamente existen algunos estudios relacionados a la limnología de ríos de la vertiente occidental de los Andes (Pascual et al., 2020) o a la composición de los bosques ribereños (MontesinosTubée et al., 2019). Sin embargo, aunque los tramos fluviales del desierto costero corresponden a las estribaciones occidentales de los Andes, las diferencias ecológicas entre ríos de flujo permanente y aquellos intermitentes son muy grandes como para poder hacer algún tipo de paralelismo entre ellos (Sarremejane et al., 2017).

Considerando que los ecosistemas áridos son espacios geográficos en los que la biodiversidad suele estar limitada en número, pero ser muy específicamente en cuanto a adaptaciones y endemismos (DeBoo et al., 2019), es posible entender la importancia regional de los espacios relictuales con abundante oferta de recursos. Por tanto, en este documento evaluamos los factores topográficos (pendiente, protección, humedad topográfica) que permiten el desarrollo de una serie de formaciones vegetales en la zona desértica del piedemonte andino-costero en las cuencas Sama, Caplina y Hospicio en la región Tacna. A partir de la 
identificación de dichas formaciones vegetales, se relaciona una serie histórica de vigor vegetal (NDVI entre 1985 y 2011) con los patrones de lluvia en las cabeceras de las mismas cuencas, y se discute sobre la posibilidad de encontrar hábitats acuáticos en dichos lugares.

\section{MATERIAL Y MÉTODOS}

\section{Área de estudio}

La región Tacna se ubica en el ecotono de los dos ecosistemas desérticos de la vertiente occidental de los Andes Sudamericanos: en el sector sur del desierto de Sechura y el sector norte del desierto de Atacama (el más árido del mundo, Becerra, 2017). El territorio regional se extiende entre las coordenadas geográficas $16^{\circ} 58^{\prime}$ y $18^{\circ} 20^{\prime}$ de latitud sur, y $69^{\circ} 28^{\prime}$ y $71^{\circ} 02^{\prime}$ de longitud oeste; dentro de dicha superficie se ubican cinco cuencas principales: Locumba, Sama, Caplina y Hospicio en la vertiente occidental, y Maure en la vertiente del Titicaca (Pino et al., 2017)

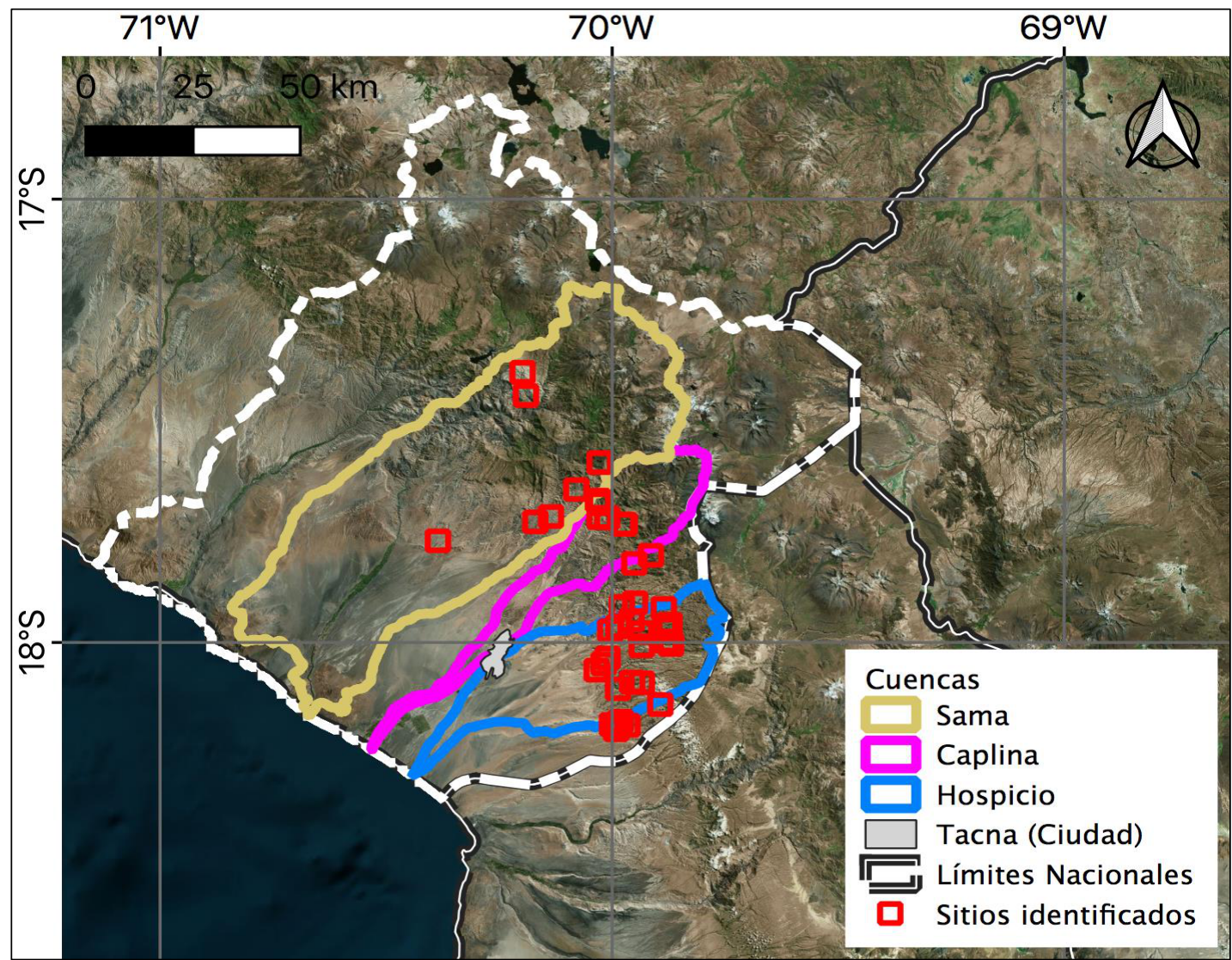

Figura 1. Mapa de ubicación de los sitios identificados (24) en el ámbito del piedemonte andino-costero de la región Tacna, cuencas de Sama, Caplina y Hospicio.

De las cinco cuencas citadas anteriormente, este trabajo de investigación se desarrolló en las cuencas de Sama, Caplina y Hospicio, debido a que en ellas se identificaron abundantes tramos fluviales intermitentes (Figura 1) y una menor intervención en los patrones hidrológicos naturales (cosa que sí ocurre en la cuenca Locumba).

La cuenca de Sama se ubica políticamente en las provincias de Tacna y Candarave, distritos de Inclán y Candarave (ANA, 2010). Altitudinalmente, la cuenca se extiende desde el nivel del mar hasta más de 3400 
m s.n.m., abarcando un área de $4591.1 \mathrm{~km}^{2}$ (ANA, 2009). La precipitación total anual varía desde $10 \mathrm{~mm}$ en la zona costera a $340 \mathrm{~mm}$ en la zona altoandina (ANA, 2010). La cuenca Caplina se ubica en la provincia de Tacna, distritos de Tacna y Pachia (ANA, 2010), su altitud varía desde 0 hasta $5690 \mathrm{~m} \mathrm{s.} \mathrm{n.} \mathrm{m.} \mathrm{en} \mathrm{la}$ Cordillera del Barroso, abarcando un área total de $908.9 \mathrm{~km}^{2}$. La cuenca presenta un clima desértico, con temperaturas promedio de $17.02^{\circ} \mathrm{C}$ y con muy escasa precipitación anual (Pino, 2013). Finalmente, la cuenca costera de Hospicio (PNUD, 2009) está ubicada principalmente en la provincia de Tacna, distrito de Calana, presenta una altitud media de $1400 \mathrm{~m} \mathrm{s.} \mathrm{n.} \mathrm{m.} \mathrm{(ANA,} \mathrm{2010)} \mathrm{y} \mathrm{un} \mathrm{área} \mathrm{de} 1347.1 \mathrm{~km}^{2}$ (ANA, 2009).

En el sector medio de las tres cuencas citadas (1 $600-2500 \mathrm{~m} \mathrm{s.} \mathrm{n.} \mathrm{m.)} \mathrm{se} \mathrm{identificaron} 24$ parches de vegetación vigorosa (vegetación ribereña) en tramos fluviales intermitentes en medio del desierto o serranía esteparia. Dentro de estos parches de vegetación ribereña, fueron analizados los valores de NDVI a lo largo de una serie histórica que cubre 25 años (1985 - 2000), dicha serie se correlacionó espacialmente con una serie de datos de precipitación extraídos de la base grillada nacional PISCO_Pm(Aybar et al., 2016).

\section{Análisis de sensores remotos}

Una de las herramientas más conocidas para el análisis de vigor vegetal y productividad primaria en base a sensores remotos es el Índice Normalizado Diferencial de Vegetación (NDVI) (Rouse et al., 1974). Este índice se basa en el comportamiento radiométrico de la vegetación en relación con la actividad fotosintética y la estructura foliar de las plantas, esta relación permite determinar la cantidad de vegetación presente y vigor vegetativo (Díaz, 2015; Meneses, 2011). La escala del índice varía entre -1 a 1, el valor cero representa la ausencia de la vegetación y los valores más cercanos a 1 representan a la vegetación más activa (Chuvieco, 1998). El cálculo del NDVI implica el uso de una fórmula con dos bandas, el infrarrojo cercano (NIR) y el rojo (RED).

$\mathrm{NDVI}=(\mathrm{NIR}-\mathrm{RED}) /(\mathrm{NIR}+\mathrm{RED})$

Donde: NIR es la reflectancia espectral del canal infrarrojo cercano (B4 en Landsat 5) y RED representa la reflectancia en el canal rojo del visible (B3 en Landsat 5) (Díaz, 2015).

Para calcular el índice NDVI se utilizaron 25 imágenes del satélite Landsat 5 (Tabla 1) descargadas del servidor de datos geográficos de USGS (https://earthexplorer.usgs.gov/). Todas las imágenes descargadas corresponden a los meses de agosto o setiembre, correspondientes a la época seca del área de estudio. El período de evaluación comprende desde el año 1985 hasta el 2011 (Tabla 1), exceptuando los años 1987 y 2002 debido a que en ellos las imágenes del área de estudio que coinciden con la época requerida son inexistentes. El uso exclusivo de imágenes Landsat 5 responde al interés de contar con información homogénea, con un registro temporal continuo, y con valores únicos de cobertura y resolución. 
Tabla 1

Imágenes Landsat 5 utilizadas en el análisis remoto

\begin{tabular}{llll}
\hline N & ID Producto & Fecha & $\%$ \\
\hline 1 & LT05_L1TP_002072_19850803_20170219_01_T1 & 1985-08-03 & 3 \\
2 & LT05_L1TP_002072_19860907_20170216_01_T1 & $1986-09-07$ & 3 \\
3 & LT05_L1TP_002072_19880928_20170206_01_T1 & $1988-09-28$ & - \\
4 & LT05_L1TP_002072_19890915_20170201_01_T1 & $1989-09-15$ & - \\
5 & LT05_L1TP_002072_19900902_20170128_01_T1 & $1990-09-02$ & 5 \\
6 & LT05_L1TP_002072_19910820_20170126_01_T1 & $1991-08-20$ & 1 \\
7 & LT05_L1TP_002072_19920907_20170123_01_T1 & $1992-09-07$ & 1 \\
8 & LT05_L1TP_002072_19930926_20170116_01_T1 & $1993-09-26$ & 9 \\
9 & LT05_L1TP_002072_19940812_20170113_01_T1 & $1994-08-12$ & 4 \\
10 & LT05_L1TP_002072_19950831_20170106_01_T1 & $1995-08-31$ & 2 \\
11 & LT05_L1TP_002072_19960801_20170103_01_T1 & $1996-08-01$ & 1 \\
12 & LT05_L1TP_002072_19970804_20161230_01_T1 & $1997-08-04$ & 5 \\
13 & LT05_L1TP_002072_19980807_20161223_01_T1 & $1998-08-07$ & 0 \\
14 & LT05_L1TP_002072_19990911_20161216_01_T1 & $1999-09-11$ & 1 \\
15 & LT05_L1TP_002072_20000929_20161213_01_T1 & $2000-09-29$ & 1 \\
16 & LT05_L1TP_002072_20010916_20161210_01_T1 & $2001-09-16$ & - \\
17 & LT05_L1TP_002072_20030821_20161204_01_T1 & $2003-08-21$ & 7 \\
18 & LT05_L1TP_002072_20040908_20161130_01_T1 & $2004-09-08$ & 7 \\
19 & LT05_L1TP_002072_20050810_20161124_01_T1 & $2005-08-10$ & 4 \\
20 & LT05_L1TP_002072_20060829_20161119_01_T1 & $2006-08-29$ & 6 \\
21 & LT05_L1TP_002072_20070816_20161111_01_T1 & $2007-08-16$ & 9 \\
22 & LT05_L1TP_002072_20080802_20161030_01_T1 & $2008-08-02$ & 0 \\
23 & LT05_L1TP_002072_20090821_20161026_01_T1 & $2009-08-21$ & 0 \\
24 & LT05_L1TP_002072_20100824_20161013_01_T1 & $2010-08-24$ & 7 \\
25 & LT05_L1TP_002072_20110912_20161006_01_T1 & $2011-09-12$ & 6 \\
\hline & & & \\
& & & \\
1
\end{tabular}

\section{Análisis morfométrico}

El análisis morfométrico del terreno permite comprender e interpretar su comportamiento morfodinámico e hidrológico, identificando los elementos del sistema que interactúan para originar procesos geomorfológicos, patrones de flujo, zonas de afloramiento y zonas de exposición y protección con respecto a factores ambientales (Méndez \& Marcucci, 2006). A partir de un modelo de elevación digital ASTER GDEM (Aster Global Digital Elevation Model, https://search.earthdata.nasa.gov/search) se calcularon tres índices morfométricos (Tabla 2): (i) el índice de protección morfométrica, (ii) el índice de humedad topográfica y (iii) la pendiente. Para estos cálculos se trabajó con la herramienta integrada SAGA en QGIS 3.12 . 
Tabla 2

Parámetros seleccionados

\begin{tabular}{|c|c|c|}
\hline Parámetro & Descripción & Fórmula \\
\hline $\begin{array}{l}\text { Protección } \\
\text { topográfica }\end{array}$ & $\begin{array}{l}\text { Determina el grado de protección que la } \\
\text { configuración morfométrica del terreno le } \\
\text { proporciona a una celda determinada (Abarca, } \\
\text { 2010). }\end{array}$ & $M P I=\frac{\sum_{J=1}^{8} \quad M A X\left(\frac{B_{i, j}}{D H_{i, j}}\right)}{8}$ \\
\hline Pendiente & $\begin{array}{l}\text { Indica el ángulo entre el plano horizontal y } \\
\text { tangencial a la superficie, refleja la tasa } \\
\text { máxima de cambio de los valores de elevación } \\
\text { (Olaya, 2009). }\end{array}$ & $P E N D I E N T E=\arctan (|\nabla \underline{Z}|)$ \\
\hline $\begin{array}{l}\text { Humedad } \\
\text { topográfica }\end{array}$ & $\begin{array}{l}\text { Cuantifica los procesos hidrológicos, indica la } \\
\text { saturación y humedad del suelo (Serafini et al., } \\
\text { 2015) }\end{array}$ & $T W I=\frac{\operatorname{Ln}([U C A])}{([S T])}$ \\
\hline
\end{tabular}

Nota: $|\nabla \underline{Z}|:$ Expresión de la gradiente en dos dimensiones $\left(\frac{\partial z}{\partial x}, \frac{\partial z}{\partial y}\right)$

\section{Análisis climatológico}

Para el análisis climatológico, se utilizó el producto grillado PISCO_Pm versión 2.0 (Aybar et al., 2016) desarrollado por el SENAMHI y disponible en el repositorio de datos SONICS (https://www.senamhi.gob.pe/?p=observacion-de-inundaciones). Para extraer y visualizar los datos de PISCO se usó R versión 3.6.1 (R Core Team, 2019) con los paquetes de raster y ncdf4 (Hijmans, 2020; Pierce, 2019). Con los datos disponibles de precipitación de época húmeda (diciembre - abril) para el territorio de las tres cuencas evaluadas (PISCO) y el valor de NDVI en los sectores de bosque ribereño ubicados en cauces de zonas desérticas, se realizó un análisis de correlación espacial (índice de Spearman) utilizando los paquetes raster, ncdf4, rgdal, xts y psych en R 3.6.1 (Bivand et al., 2019; Ryan \& Ulrich, 2020; Revelle, 2020). Este análisis de correlación espacial ayuda a inferir la relación que potencialmente existiría entre la precipitación, la infiltración y la recarga de acuíferos, con la productividad primaria en los bosques ribereños identificados en las zonas desérticas.

\section{RESULTADOS}

\section{NDVI}

Los valores obtenidos para el NDVI en los bosques ribereños identificados presentan una distribución relativamente homogénea con valores positivos entre 0.1 y 0.4 unidades, lo que indica un vigor vegetal medio a alto probablemente asociado a zonas con disponibilidad de recursos hídricos. Existen ligeras diferencias atribuibles a variabilidad ambiental interanual, como años con diferentes niveles de precipitación o registros térmicos, lo que podría afectar a la disponibilidad de agua por diferencias en la recarga del acuífero regional o distintos grados de demanda por evapotranspiración debido a la variabilidad térmica. A modo de ejemplo: los años 1985, 2000 y 2005 presentan una distribución con mayor amplitud en los valores de NDVI con alta densidad, registrándose la máxima densidad de observaciones en un valor alrededor de 0.25 unidades. Por otro lado, los años 1990, 1995 y 2010 presentan una distribución de valores con menor amplitud y con un pico de densidad entre 0.1 y 0.15 unidades, lo que indicaría condiciones de menor vigor vegetal. Estas diferencias pueden presentarse como un patrón cíclico dependiente de la 


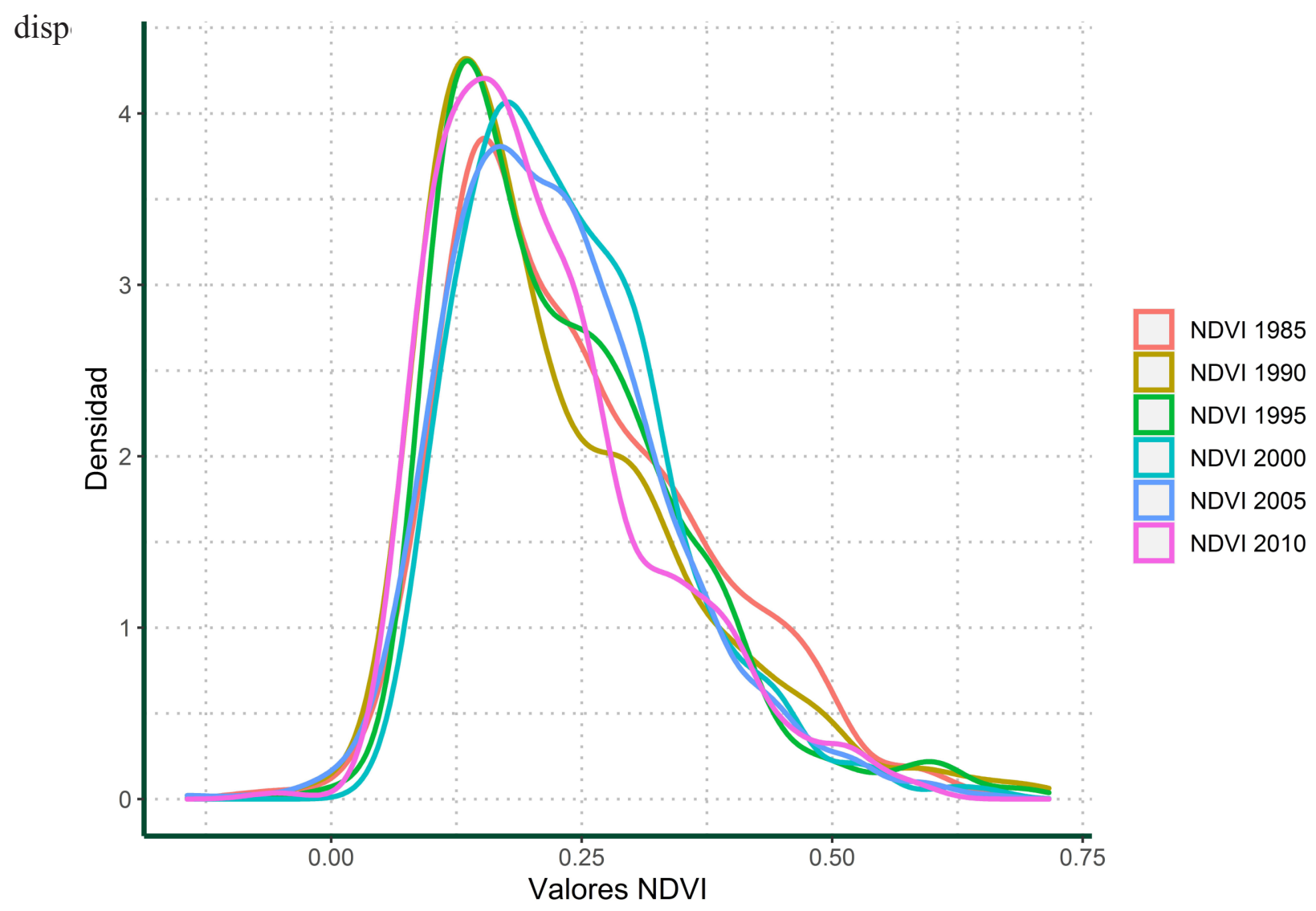

Figura 2. Curvas de densidad de los valores de NDVI obtenidos en los años 1985-2010

\section{Índices morfométricos}

El índice de protección morfométrica muestra una mayor concentración de valores entre 0.16 y 0.30 unidades, indicando que el área de muestreo se encuentra parcialmente protegida del viento e insolación, en zonas donde la capacidad de protección depende del ancho del valle y su orientación. Por otro lado, los valores de la pendiente van desde $1^{\circ}$ hasta $29^{\circ}$ continuamente, se presentan además valores dispersos entre $29^{\circ}$ y $41^{\circ}$, y se muestra una mayor concentración entre $5^{\circ}$ y $15^{\circ}$; en general, el $50 \%$ de los datos son mayores de $9^{\circ} \mathrm{y}$, teniendo en cuenta que hay mayor concentración hasta los $15^{\circ}$, la pendiente se interpreta como mediana a baja, correspondiente a la zona de fondo de valle fluvial. Finalmente, la humedad topográfica muestra valores altos, entre 12 a 18 unidades, esto indica que el área de estudio se encuentra cerca de aguas subterráneas y es potencialmente un lugar de afloramiento ya que se encuentra en el fondo del valle (Figura $3)$. 

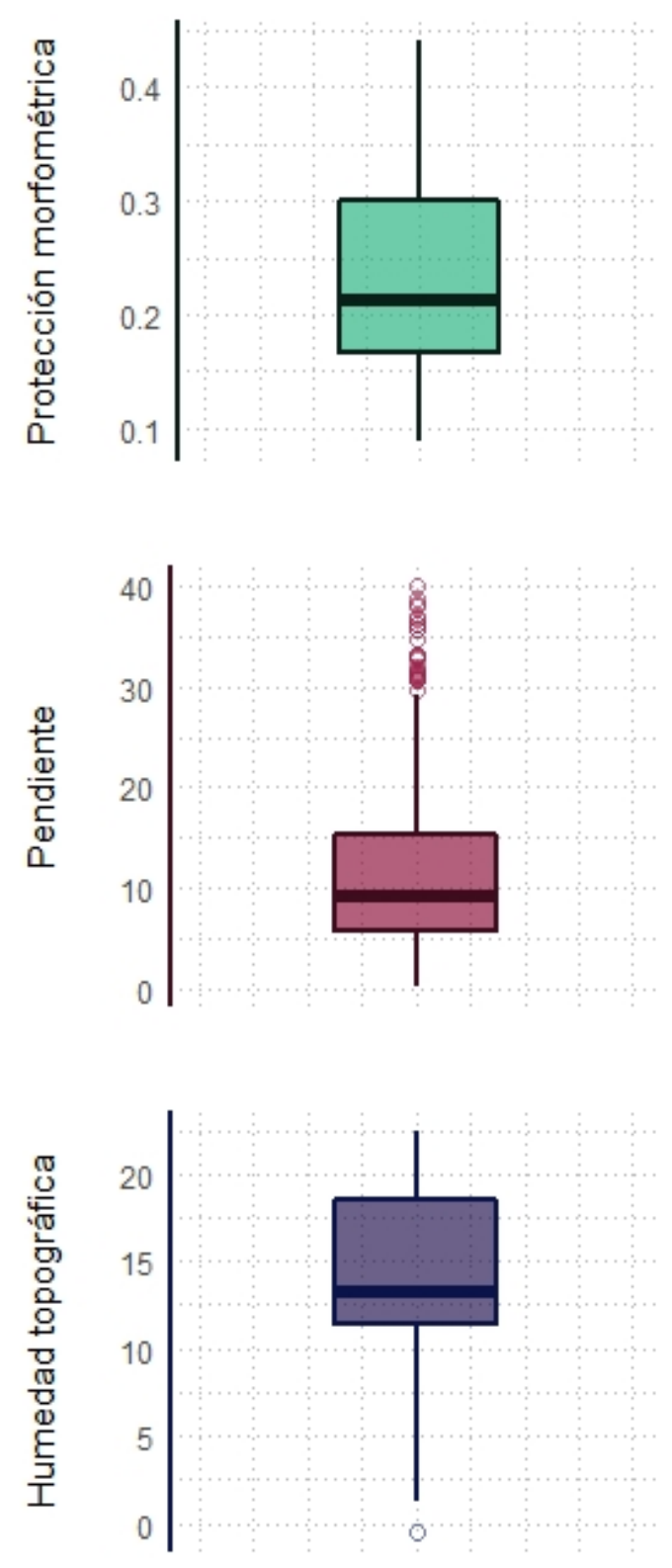

Figura 3. Índices morfométricos

\section{Precipitación}

Para este análisis se extrajeron los resultados de precipitación mensual ( $\mathrm{mm} / \mathrm{mes}$ de la grilla PISCO_Pm) en el período comprendido entre 1981 a 2016 en cuatro puntos aleatorios dentro del ámbito de estudio, los cuales representan diferentes pisos altitudinales (Figura 4). A $414 \mathrm{~m} \mathrm{s.} \mathrm{n.} \mathrm{m.,} \mathrm{en} \mathrm{el} \mathrm{piedemonte} \mathrm{andino-}$ costero, la precipitación obtiene sus registros menores con picos por debajo de $25 \mathrm{~mm} / \mathrm{mes}$. A $1152 \mathrm{~m} \mathrm{s.} \mathrm{n}$. m., en la parte inferior de la serranía esteparia, el registro de precipitación presenta valores muy frecuentes de ausencia total de precipitación y máximas alrededor de $25 \mathrm{~mm} / \mathrm{mes}$. A $2419 \mathrm{~m} \mathrm{~s}$. n. m., el gráfico se observa con picos más amplios que pueden alcanzar e incluso sobrepasar los $50 \mathrm{~mm} / \mathrm{mes}$. Finalmente, a una altura de $4661 \mathrm{~m} \mathrm{~s}$. n. m. se aprecian los valores más altos de precipitación, pudiendo llegar a registrarse entre 150 y $200 \mathrm{~mm} / \mathrm{mes}$ de lluvia en la época húmeda. Esta distribución altitudinal de la precipitación coincide con lo previsto por la regionalización de Rau et al. (2017) para la vertiente del Pacífico en el 
territorio peruano.

Precipitación (mm/Mes)
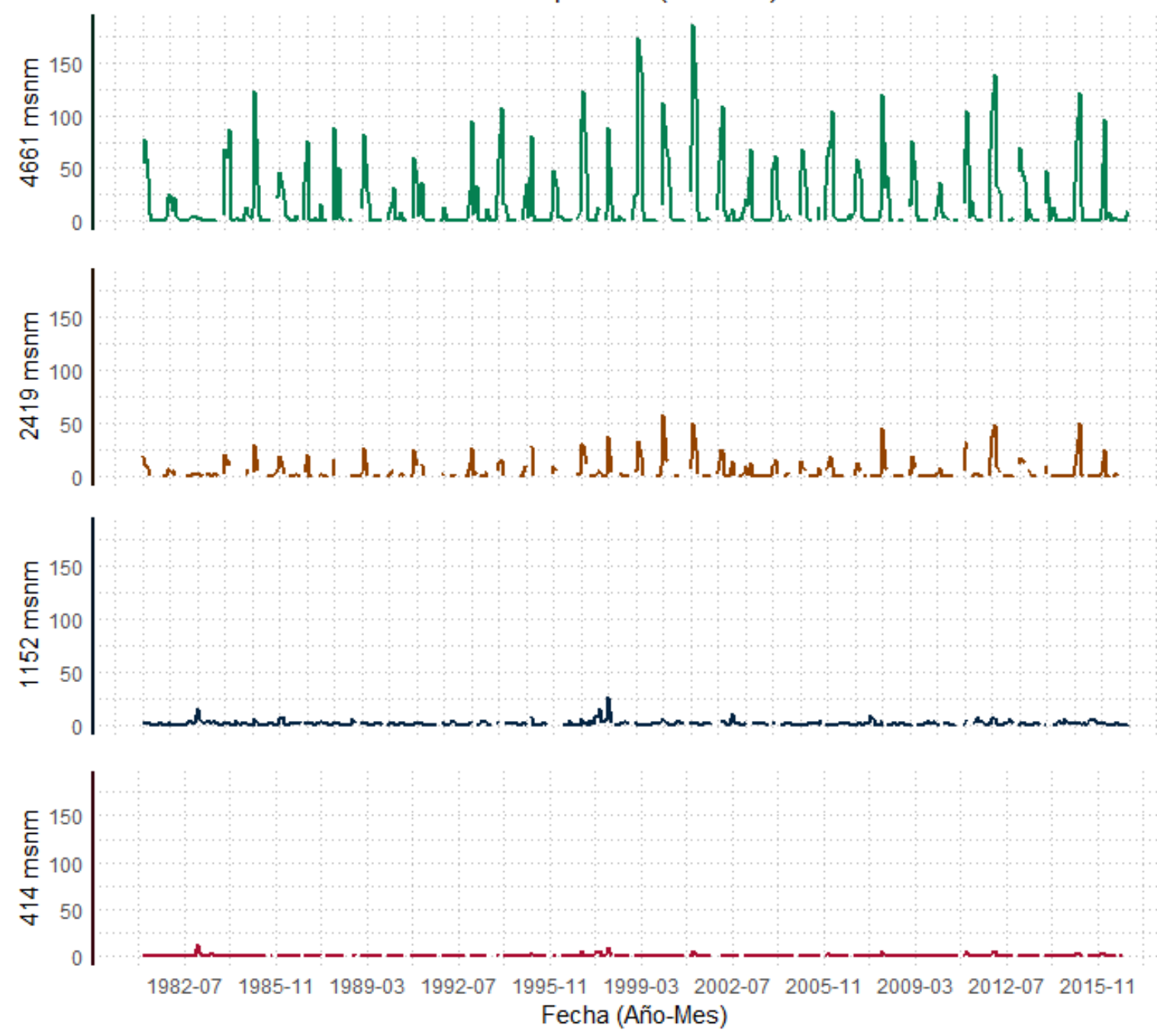

Figura 4. Precipitación por piso altitudinal

\section{Correlación espacial entre NDVI y precipitación}

La correlación espacial entre la precipitación y el NDVI en los bosques ribereños identificados como objeto de estudio (Figura 5), se realizó considerando cuatro escenarios de demora de respuesta: A respuesta a la precipitación en el mismo año hidrológico, B respuesta a la precipitación en el año hidrológico anterior, C respuesta a la precipitación dos años antes y $\mathrm{D}$ respuesta a la precipitación tres años antes. 
$\mathbf{A}$

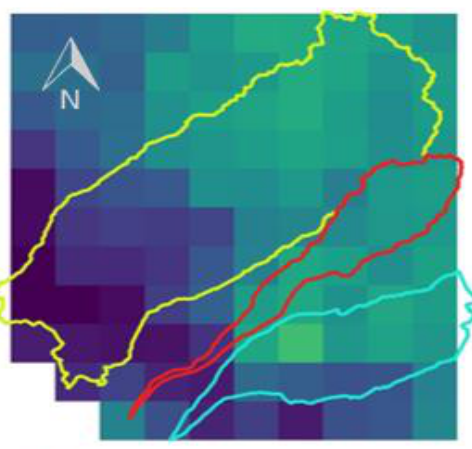

C

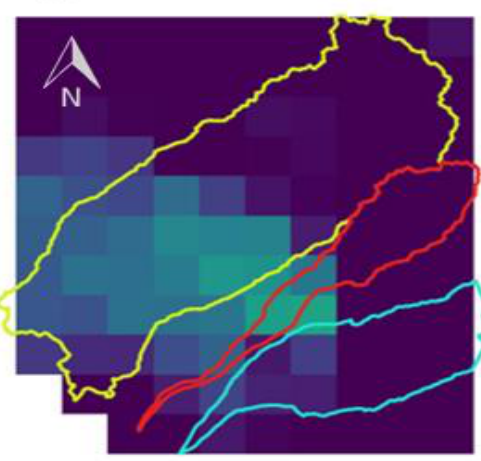

B

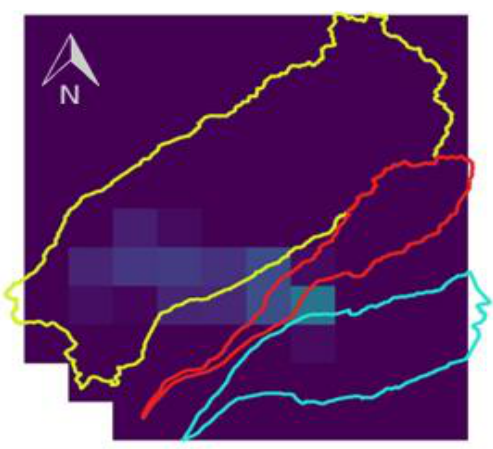

D

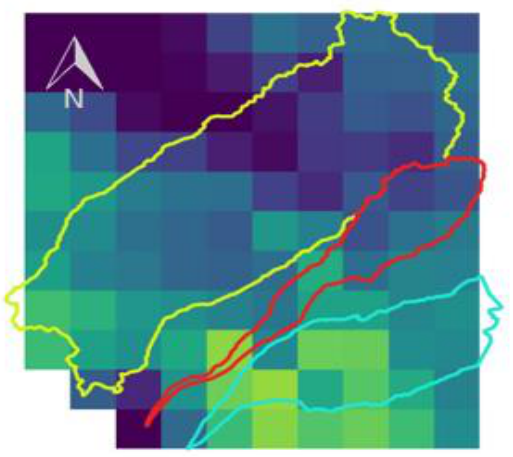

Correlación

0.60

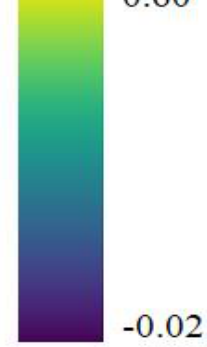

$-0.02$

\section{Cuencas}

Sama

Caplina

Hospicio

Figura 5. Correlación espacial entre precipitación (a nivel de cuenca) y NDVI (en bosques ribereños)

Los escenarios A y C presentan los valores más altos de correlación espacial. Al analizar estos patrones, es posible apreciar que en el escenario A existe una mayor influencia de precipitaciones en los sectores altos de la cuenca, donde se concentra la mayor cantidad de precipitación; mientras que en el escenario D, las correlaciones más altas provienen de las zonas medias o bajas. Asumiendo que los parches de vegetación ribereña identificados son principalmente ecosistemas dependientes de agua subterránea, es posible inferir que el escenario A explica mejor los patrones de variabilidad en vigor vegetal. De esta manera, la cantidad de precipitación ocurrida en las zonas altas de la cuenca contribuye a la recarga del acuífero regional, y como respuesta a una mayor o menor disponibilidad de recursos hídricos, existe una variación directamente correlacionada con el valor de NDVI en los bosques ribereños.

\section{DISCUSIÓN}

Los valores de NDVI obtenidos muestran que el vigor vegetal está correlacionado positivamente con la precipitación en la parte alta de la cuenca, y dicha precipitación constituye la principal fuente de recarga del acuífero regional. Estos resultados son concordantes con los de Hao et al., (2012), quienes evaluaron la relación entre precipitación y temperatura con el NDVI en bosques y pastizales en la cuenca alta del río Amarillo (China), encontrando que existe una correlación positiva entre el vigor y ambos parámetros ambientales. De forma similar, Fu \& Burgher (2015) encuentran que - en zonas áridas - la disponibilidad de agua en el acuífero superficial es fundamental para mantener el vigor vegetal en bosques ribereños (como los que evaluamos) en la temporada seca. Schmidt \& Karnieli (2000) realizaron mediciones espectrales de diferentes componentes de vegetación en zonas lluviosas, encontrando que el NDVI se reduce al pasar de la época húmeda a la seca debido a la desaparición de herbáceas anuales, las costras biogénicas también presentan un espectro de vegetación verde durante las temporadas de lluvia, ambos factores muestran la importancia de la disponibilidad de agua para obtener una respuesta espectral vigorosa. Esto explicaría nuestros resultados con variaciones en el NDVI relacionadas a los patrones de recarga del acuífero regional. 
Chuai et al. (2012) realizaron un análisis de correlación entre el NDVI y variables climáticas para observar respuesta al cambio climático en zonas áridas y semi-áridas, encontrando generalmente una correlación negativa con la temperatura y positiva con la precipitación. Zhang \& Guo (2008) mencionan que el tiempo de precipitación determina la vitalidad de los ecosistemas semi-áridos, y que la descarga de acuíferos someros es una fuente importante de humedad del suelo en estos ecosistemas (He et al., 2007). Las variaciones espacio-temporales del valor medio de precipitación podrían ser interpretadas como un indicador de desertificación (Prince et al., 1998); sin embargo, nuestros datos no muestran variaciones en cuanto a las tendencias temporales de la precipitación total anual. En Argentina, Gaitán et al. (2014) encontraron que la proporción de la precipitación que cae en primavera-verano se relacionó positivamente con la cobertura de pastos, de forma similar nuestro estudio muestra que el NDVI presenta una relación positiva con respecto a la precipitación en la zona de recarga de acuíferos de la cuenca.

Los valores medios del índice de protección morfométrica se relacionan con factores como la exposición al viento, insolación y radiación solar (Toivonen et al., 2017), además, proporciona sombra reduciendo la tasa de evaporación; por tanto, la combinación de zonas de descarga y zonas de protección permiten el desarrollo de vegetación. En concordancia, en nuestro análisis los bosques ribereños se desarrollan en valles estrechos con altos niveles de protección morfométrica. Los valores que obtuvimos para la pendiente en este trabajo pueden interpretarse como medios a bajos, esto es relevante, ya que la pendiente controla factores como la disponibilidad y espesor de suelos y su distribución (Toivonen et al., 2017). Teniendo en cuenta la pendiente, las propiedades del suelo varían: las áreas más bajas acumulan corrientes de agua y sedimentos derivados de las áreas circundantes altas. Estos valores bajos o medios de pendiente se relacionan inversamente con los valores obtenidos para el índice de humedad topográfica: nuestros resultados muestran que la mayor parte de parches de vegetación ribereña ubicados en el desierto de Tacna se ubican en zonas de fondo de quebrada donde la probabilidad de acceder al acuífero (lo que mide el índice de humedad topográfica) es alta y la pendiente baja. Según Gaitan et al. (2014) es probable que en los ecosistemas áridos y semi-áridos disminuya el vigor vegetal, estos resultados nos muestran el impacto y relación que tiene el clima sobre el funcionamiento del ecosistema árido (Knapp et al., 2002)

En los ecosistemas áridos la vegetación evita, resiste o tolera la desecación (Lyons \& Breidenbach, 1981), esto es una capacidad generalizada de todas las plantas adaptadas a estas condiciones (Rascio \& La Rocca, 2005). El agua subterránea en las regiones áridas o semi-áridas juega un papel crucial en el mantenimiento de ecosistemas terrestres nativos. Su profundidad controla la disponibilidad de agua para la vegetación (Eamus et al., 2016), además, conserva los ecosistemas terrestres dependientes de ella. Las alteraciones causarán fluctuaciones en la profundidad del agua subterránea, que es el factor ambiental clave (Shang et al., 2016). En estas regiones, especialmente durante los períodos secos con escasa precipitación, el agua subterránea tiene un efecto esencial sobre el contenido de humedad del suelo que influye en la estabilidad ecológica local (Huang et al., 2019). Las zonas áridas, semi-áridas y subhúmedas secas reciben apenas el 2 $\%$ de la precipitación pluvial anual que cae en el país.

En nuestra zona de estudio se observó que la vegetación presenta alto vigor a pesar de encontrarse en un ecosistema árido, posiblemente esto se deba a la descarga de aguas subterráneas. Esto se refuerza por el análisis de los datos morfológicos, ya que los valores nos muestran que el bosque se desarrolla en fondos de valle, donde es más probable que el agua se descargue. Además, el bosque se desarrolla en zonas que presentan un valor alto en protección morfológica, lo que asegura que el agua no se evapore con facilidad, pudiendo deducirse que se trata de ecosistemas dependientes de agua subterránea (Kløve et al., 2011).

Si las condiciones de cobertura y protección son suficientes, es altamente probable que en estos valles estrechos se encuentren pequeños tramos con flujo de agua superficial albergando comunidades acuáticas aisladas. Estas comunidades constituirían reservorios importantes de diversidad para una región árida como la nuestra (Mossop et al., 2015, 2017), pero se cuenta con muy poca información sobre ellos lo que limita su gestión sostenible (O'Brien, 2019). La conectividad esporádica pero recurrente entre diferentes tramos intermitentes como los descritos antes, es un elemento fundamental para la renovación del acervo genético 
local (Goodrich et al., 2018). Sin embargo, la periodicidad de esta conectividad podría alterarse en el contexto del cambio climático trayendo consigo variaciones en las características generales del medio, en la carga genética de la comunidad y en la sostenibilidad de todo el sistema.

Nuestro estudio cuenta con dos limitaciones importantes que mencionar: (i) esta fase del trabajo se basa en información satelital y (ii) en la discusión se plantean algunas hipótesis que dependen de asunciones generales con respecto a la ecología de ríos intermitentes. Para el conocimiento de los autores, no se han publicado (y presumiblemente tampoco desarrollado) estudios acerca de la ecología de tramos intermitentes en la región Tacna ni en el resto del territorio nacional. Considerando dichas limitaciones, se plantea este trabajo como un objeto de discusión inicial sobre ecosistemas que tienen un gran potencial como refugios de biodiversidad, en particular en regiones áridas donde la disponibilidad de agua resulta un elemento limitante fundamental. Una limitación adicional, más operativa, resulta de la resolución de las imágenes utilizadas (Landsat 5, $30 \times 30 \mathrm{~m}$ ) comparadas con otras también disponibles (Sentinel p.ej.); sin embargo, se eligieron imágenes Landsat 5 debido a la amplia base de datos que presenta en los años de interés.

\section{CONCLUSIONES}

De acuerdo a los resultados obtenidos, los relictos de bosque ribereño que inesperadamente aparecen en medio del desierto de Tacna presentan un alto vigor vegetal debido a que serían dependientes de agua subterránea. Esta agua subterránea se descarga regularmente en las zonas intermedias de la cuenca donde una serie de factores morfológicos como la protección (estrechez del valle) y la pendiente (fondos) reducen sus pérdidas por evaporación y la mantienen disponible para el ecosistema. La zona de recarga del acuífero correspondería a las cabeceras de cuenca y la respuesta más sólida de la vegetación durante la temporada seca se da con respecto a los valores de precipitación en la época de lluvias del mismo año, esto indicaría que el periodo de tránsito de los recursos hídricos subterráneos entre la zona de recarga y la cuenca media sería menor a un año.

Debido a la presencia de acuíferos someros y manantiales en el sector medio de la cuenca, en combinación con una serie de factores morfológicos que limitan su evaporación, existe una gran probabilidad de presencia de tramos con flujo de agua superficial. En estos tramos inconexos de arroyos intermitentes, los organismos acuáticos constituyen poblaciones aisladas, pero representan también un elemento fundamental (e inesperado) de la diversidad biológica regional. Describir y entender las dinámicas de esta comunidad resulta urgente para incorporarla en los planes regionales de conservación de la diversidad biológica.

\section{AGRADECIMIENTOS}

Este trabajo constituye el primer proyecto desarrollado por el Semillero de Limnología de la Facultad de Ciencias de la Universidad Nacional Jorge Basadre Grohmann. La idea original del artículo se gestó en base a una serie de discusiones y prácticas desarrolladas en las instalaciones del Herbario Takana (TKA) con el profesor Eduardo Oyague; por ello, expresamos un profundo agradecimiento a su persona por su mentoria en el desarrollo del artículo. Al mismo tiempo, los integrantes del proyecto también desean agradecer el soporte brindado por la profesora Liduvina Sulca. Finalmente, deseamos agradecer los comentarios de un árbitro anónimo que nos ayudaron a mejorar sustancialmente el artículo.

\section{REFERENCIAS}

Abarca, O. (2010). Desarrollo de un modelo de geoprocesamiento para la valoración productiva y tributaria de tierras agrícolas en Venezuela. Universidad Politécnica de Madrid.

ANA. (2009). Demarcación y delimitación de las Autoridades administrativas del agua. Ministerio de Agricultura. 
ANA. (2010). Estudio evaluación de recursos hídricos en cuencas de los ríos Locumba y Sama Tacna. Ministerio de Agricultura, I.

Arnold, S., Attinger, S., Frank, K., Baxter, P., Possingham, H., \& Hildebrandt, A. (2016). Ecosystem Management Along Ephemeral Rivers: Trading Off Socio-Economic Water Supply and Vegetation Conservation under Flood Regime Uncertainty. River Research and Applications, 32(3), 219-233.

Aybar, C., Lavado, W., Huerta, A., Fernández, C., Vega, F., Sabino, E., \& Felipe, O. (2016). Uso del producto grillado PISCO de precipitación en estudios, investigaciones y sistemas operacionales de monitoreo y pronóstico hidrometeorológico. senamhi, 1-22.

Becerra, G. (2017). Procesos eco territoriales en la ocupación del desierto sur occidental del Perú. Cienciay Desarrollo, 16(2), 85-99.

Bivand, R., Keitt, T., \& Barry, R. (2019). rgdal: Bindings for the «Geospatial» Data Abstraction Library. Recuperado de https://cran.r-project.org/package $=$ rgdal

Bunn, S., Davies, P., \& Winning, M. (2003). Sources of organic carbon supporting the food web of an arid zone floodplain river. Freshwater Biology, 48(4), 619-635.

Bunn, S., Thoms, M., Hamilton, S., \& Capon, S. (2006). Flow variability in dryland rivers: boom, bust and the bits in between. River Research and Applications, 22(2), 179-186.

Chuai, X., Huang, X., Wang, W., \& Bao, G. (2012). NDVI, temperature and precipitation changes and their relationships with different vegetation types during 1998-2007 in Inner Mongolia, China. International Journal of Climatology, 33(7), 1696-1706.

Chuvieco, E. (1998). El factor temporal en teledetección: evolución fenomenológica y análisis de cambios. Revista de teledetección: Revista de la Asociación Española de Teledetección, (10), 4.

Datry, T., Boulton, A., Bonada, N., Fritz, K., Leigh, C., Sauquet, E., Tockner, K., Hugueny, B., \& Dahm, C. (2017). Flow intermittence and ecosystem services in rivers of the Anthropocene. Atmospheric Environment, 23(1994), 248-258.

DeBoo, M., Murphy, N., Austin, A., Watts, C., \& Guzik, M. (2019). Arid zone island hopping: the impact of dispersal on endemism in hydraenid beetles (Coleoptera: Hydraenidae) found in isolated desert springs. Austral Entomology, 58(4), 886-896.

Díaz, J. (2015). Estudio de Índices de vegetación a partir de imágenes aéreas tomadas desde UAS/RPASy aplicaciones de estos a la agricultura de precisión. Universidad Complutense De Madrid.

Eamus, D., Fu, B., Springer, A., \& Stevens, L. (2016). Groundwater Dependent Ecosystems: Classification, Identification Techniques and Threats. En Jakeman, A., Barreteau, O., Hunt, R., Rinaudo, J., \& Ross, A. (Eds.), Integrated Groundwater Management: Concepts, Approaches and Challenges (pp. 313-346). Cham: Springer International Publishing. https://doi.org/10.1007/978-3-319-23576913

Fu, B., \& Burgher, I. (2015). Riparian vegetation NDVI dynamics and its relationship with climate, surface water and groundwater. Journal of Arid Environments, 113, 59-68.

Gaitán, J., Oliva, G., Bran, D., Maestre, F., Aguiar, M., Jobbágy, E., Buono, G., Ferrante, D., Nakamatsu. V. Ciari, G., Salomone, J., \& Massara, V. (2014). Vegetation structure is as important as climate for explaining ecosystem function across patagonian rangelands. Journal of Ecology, 102(6), 1419-1428.

Goodrich, D., Kepner, W., Levick, L., \& Wigington, P. (2018). Southwestern Intermittent and Ephemeral Stream Connectivity. Journal of the American Water Resources Association, 54(2), 400-422.

Hao, F., Zhang, X., Ouyang, W., Skidmore, A., \& Toxopeus, A. (2012). Vegetation NDVI Linked to Temperature and Precipitation in the Upper Catchments of Yellow River. Environmental Modeling \& Assessment, 17.

He, Y., Guo, X., \& Si, B. C. (2007). Detecting grassland spatial variation by a wavelet approach. International Journal of Remote Sensing, 28(7), 1527-1545.

Hijmans, R. (2020). Raster: Geographic Data Analysis and Modeling. Recuperado de https://cran.rproject.org/package $=$ raster 
Huang, F., Zhang, Y., Zhang, D., \& Chen, X. (2019). Environmental groundwater depth for groundwaterdependent terrestrial ecosystems in arid/semiarid regions: A review. International Journal of Environmental Research and Public Health, 16(5).

Kaletová, T., Loures, L., Castanho, R. A., Aydin, E., da Gama, J. T., Loures, A., \& Truchy, A. (2019). Relevance of intermittent rivers and streams in agricultural landscape and their impact on provided ecosystem services - a mediterranean case study. International Journal of Environmental Research and Public Health, 16(15).

Kalogianni, E., Vourka, A., Karaouzas, I., Vardakas, L., Laschou, S., \& Skoulikidis, N. (2017). Combined effects of water stress and pollution on macroinvertebrate and fish assemblages in a Mediterranean intermittent river. Science of The Total Environment, 603-604, 639-650.

Kløve, B., Allan, A., Bertrand, G., Druzynska, E., Ertürk, A., Goldscheider, N., Henry, S., Karakaya, N., Karjalainen, T., Koundouri, P., Kupfersberger, H., Kvœrner, J., Lundberg, A., \& Muotka, T. (2011). Groundwater dependent ecosystems. Part II. Ecosystem services and management in Europe under risk of climate change and land use intensification. Environmental Science and Policy, 14(7), 782-793.

Knapp, A., Fay, P., Blair, J., Collins, S., Smith, M., Carlisle, J. Harper, C., Danner, B., Lett, M., \& McCarron, J. (2002). Rainfall variability, carbon cycling, and plant species diversity in a mesic grassland. Science, 298(5601), 2202-2205.

Leigh, C., Boulton, A. Courtwright, J., Fritz, K., May, C., Walker, R., \& Datry, T. (2016). Ecological research and management of intermittent rivers: an historical review and future directions. Freshwater Biology, 61(8), 1181-1199.

Lyons, J., \& Breidenbach, R. (1981). Book Review:Responses of Plants to Environmental Stresses. J. Levitt, T. T. Kozlowski. The Quarterly Review of Biology.

Méndez, W., \& Marcucci, E. (2006). Análisis morfométrico de la microcuenca de la quebrada Curucutí, estado Vargas-Venezuela. Revista Geográfica Venezolana, 47(1), 29-55.

Meneses, C. (2011). El índice normalizado diferencial de la vegetación como indicador de la degradación del bosque (Vol.62)

Montesinos-Tubée, D., Núñez del Prado, H., Toni, B., Álvarez, E., Borgoño, A., Zegarra, J., Gutiérrez, G., Maldonado, M., Rodríguez, M., Riveros, G., \& Guillén, D. (2019). Diversidad florística, comunidades vegetales y propuestas de conservación del monte ribereño en el río Chili (Arequipa, Perú). Arnaldoa, 26(1), 97-130.

Mossop, K., Adams, M., Unmack, P., Smith, K., Wong, B., \& Chapple, D. (2015). Dispersal in the desert: Ephemeral water drives connectivity and phylogeography of an arid-adapted fish. Journal of Biogeography, 42(12), 2374-2388.

Mossop, K., Moran, N., Chapple, D., \& Wong, B. (2017). Connectivity and habitat type shape divergent dispersal behavior in a desert-dwelling fish. Landscape Ecology, 32(5), 1065-1078. Springer Netherlands.

Olaya, V. (2009). Chapter 6 Basic Land-Surface Parameters. In T. Hengl y H. I. R. Reuter (Eds.), Geomorphometry, 33, 141-169.

O'Brien, G., Mathew, R., Hanzen, C., Dlamini, V., Petersen, R., Diedericks, G., \& Burnett, M. (2019). River connectivity and fish migration considerations in the management of multiple stressors in South Africa. Marine and Freshwater Research, 70(9), 1254-1264. https://doi.org/10.1071/MF19183

Pascual, G., Iannacone, J., \& Alvariño, L. (2020). Macroinvertebrados bentónicos y ensayos toxicológicos para evaluar la calidad del agua y del sedimento del río Rímac, Lima, Perú. Revista de Investigaciones Veterinarias del Perú, 30(4), 1421-1442.

Pierce, D. (2019). Ncdf4: Interface to Unidata netCDF (Version 4 or Earlier) Format Data Files. Recuperado dehttps://cran.r-project.org/package=ncdf4

Pino, C. (2013). Caracterización hidrogeomorfológica de la cuenca del río Caplina - Tacna (tesis de pregrado). Universidad Nacional Jorge Basadre Grohmann. 
Pino, E., Tacora, P., Steenken, A., Alfaro, L., Valle, A., Chávarri, E., Ascencios, D., \& Mejía, J. (2017). Efecto de las características ambientales y geológicas sobre la calidad del agua en la cuenca del río Caplina, Tacna, Perú. Tecnología y Ciencias del Agua, 8(6), 77-99.

PNUD. (2009). Población : las cuencas articulan la convivencia. En Informe sobre Desarrollo Humano Perú 2009: Por una densidad del Estado al servicio de la gente.

Prince, S., Brown De Colstoun, E., \& Kravitz, L. (1998). Evidence from rain-use efficiencies does not indicate extensive Sahelian desertification. Global Change Biology, 4(4), 359-374.

Rascio, N., \& La Rocca, N. (2005). Resurrection plants: The puzzle of surviving extreme vegetative desiccation. Critical Reviews in Plant Sciences, 24(3), 209-225.

R Core Team. (2019). R: A Language and Environment for Statistical Computing. Vienna, Austria: $R$ Foundation for Statistical Computing. Recuperado de https://www.r-project.org/

Revelle, W. (2020). Psych: Procedures for Psychological, Psychometric, and Personality Research. Evanston, Illinois: Northwestern University. Recuperado de https://cran.rproject.org $/$ package $=$ psych

Rouse J., Haas, R., Schell, J., \& Deering, D. (1974). Monitoring Vegetation Systems in the Great Plains with Erts. NASA Special Publication, 351, p. 309.

Ryan, J., \& Ulrich, J. (2020). Xts: eXtensible Time Series. Recuperado de https://cran.rproject.org/package $=$ xts

Schmidt, H., \& Karnieli, A. (2000). Remote sensing of the seasonal variability of vegetation in a semi-arid environment. Journal of Arid Environments, 45(1), 43-59.

Sarremejane, R., Cañedo-Argüelles, M., Prat, N., Mykrä, H., Muotka, T., \& Bonada, N. (2017). Do metacommunities vary through time? Intermittent rivers as model systems. Journal of Biogeography, 44(12), 2752-2763.

Serafini, M., Fea, M., Di Franco, L., Cuello, A., Antes, M., Villanueva, S., Sione, W., González, F., \& Redondo, F. (2015). Análisis de parámetros morfométricos y su relación con condiciones de vulnerabilidad en la ecorregión de las Yungas, Argentina. Ciencias Espaciales, 8(1), 174-189.

Shang, H., Wang, W., Dai, Z., Duan, L., Zhao, Y., \& Zhang, J. (2016). An ecology-oriented exploitation mode of groundwater resources in the northern Tianshan Mountains, China. Journal of Hydrology, 543,386-394. http://dx.doi.org/10.1016/j.jhydrol.2016.10.012

Stanley, E., Fisher, S., \& Grimm, N. (1997). Ecosystem Expansion and Contraction in Streams. BioScience, 47(7), 427-435.

Stromberg, J., \& Merritt, D. (2016). Riparian plant guilds of ephemeral, intermittent and perennial rivers. Freshwater Biology, 61(8), 1259-1275.

Toivonen, J., Gonzales-Inca, C., Bader, M., Ruokolainen, K., \& Kessler, M. (2017). Elevational shifts in the topographic position of polylepis forest stands in the Andes of Southern Peru. Forests, 9(1), 1-10.

Von Schiller, D., Bernal, S., Dahm, C., \& Martí, E. (2017). Nutrient and Organic Matter Dynamics in Intermittent Rivers and Ephemeral Streams. Intermittent Rivers and Ephemeral Streams: Ecology and Management, 135-160.

Wang, L., \& D'Odorico, P. (2008). Decomposition and Mineralization. Encyclopedia of Ecology, 5, $838-844$.

Zhang, C., \& Guo, X. (2008). Monitoring northern mixed prairie health using broadband satellite imagery. International Journal of Remote Sensing, 29(8), 2257-2271. 